\title{
Infecção pelo Papilomavirus Humano em Adolescentes: Relação com 0 Método Anticoncepcional, Gravidez, Fumo e Achados Citológicos
}

\author{
Human Papillomavirus Infection in Adolescents: Relation to Contraceptive Method, Pregnancy, \\ Smoking, and Cytologic Findings \\ Eddie Fernando Candido Murta ${ }^{1}$, Maria Azniv Hazarabedian de Souza ${ }^{1}$, \\ Sheila Jorge Adad $^{2}$, Edward Araújo Júnior ${ }^{1}$
}

\begin{abstract}
RESUMO
Objetivo: avaliar a influência da gravidez, do hábito de fumar, do método anticoncepcional e quais os achados citológicos mais freqüentes em adolescentes com infecção pelo HPV.

Métodos: foram analisadas retrospectivamente 54.985 citologias de pacientes atendidas entre julho de 1993 e dezembro de 1998. Deste total, 6.498 exames $(11,8 \%)$ eram de pacientes com idade inferior a 20 anos, sendo que 326 (5,9\%) apresentavam sinais citológicos de infecção por HPV, associada ou não a neoplasia intra-epitelial cervical (NIC) grau I. O grupo controle foi composto por 333 pacientes na mesma faixa etária, sem sinais citológicos de infecção por HPV.

Resultados: a infecção pelo HPV foi mais freqüente nas adolescentes que fazem uso de anticoncepcionais orais $(16,9 \%$ versus $13,8 \%, p<0,01)$ e que apresentam o achado citológico de clue cells $(22,4 \%$ versus $14,7 \%, p<0,001)$. Os casais usuários de condom tiveram menor freqüência de infecção pelo $H P V(0 \%$ versus $2,1 \%, p<0,01)$. A diferença do número de gestantes $(41,1 \%$ versus $44,1 \%)$ e de fumantes $(21,8 \%$ versus $16,5 \%)$ não foi estatisticamente significante.

Conclusões: a infecção pelo HPV é mais freqüente em mulheres adolescentes que fazem uso de anticoncepcionais orais e que apresentam achado citológico de "clue cells". Os casais usuários de condom tiveram menor incidência de infecção pelo HPV. A gestação e o hábito de fumar não influenciaram na incidência da infecção pelo HPV.
\end{abstract}

PALAVRAS-CHAVE: Papilomavirus humano. Adolescentes. Citologia cérvico-vaginal. Contracepção.

\section{Introdução}

$\mathrm{Na}$ adolescência a atividade biológica cervical está em nível máximo. Nesta fase, a replicação celular e substâncias presentes no meio cervical facilitam a infecção por papilomavírus humano $(\mathrm{HPV})^{1}$. Após a adolescência, a freqüência da infecção pelo HPV nas mulheres dimi-

${ }^{1}$ Disciplina de Ginecologia e Obstetrícia da Faculdade de Medicina do Triângulo Mineiro.

${ }^{2}$ Disciplina de Patologia Especial da Faculdade de Medicina do Triângulo Mineiro.

Correspondência:

Eddie Fernando Candido Murta

Rua: Alfén Paixão, 170, Apto. 202

38060-230 - Uberaba - MG

Tel: (34) 3318-5326, Fax: (34) 3333-8710 - 3318-5342

e-mail: eddiemurta@mednet.com.br nui com a idade $^{2}$.

Independentemente da faixa etária, fatores relacionados à maior freqüência de infecção pelo HPV, como a gravidez, hábito de fumar, método anticoncepcional e infecções genitais, têm sido estudados. Alguns autores demonstraram maior incidência de infecção por HPV em gestantes, sugerindo ser a gestação um fator de risco independente para infecção por HPV ${ }^{3-5}$. No entanto, outros autores descrevem incidência igual ou menor em mulheres gestantes em relação às não-gestantes ${ }^{6-8}$.

Em relação aos métodos contraceptivos, o uso de anticoncepcional oral (ACO) parece ser um fator de risco isolado para aumentar a incidência de infecção por HPV. Este aumento é explicado por alterações hormonais que levariam a imunomodulação ${ }^{9} \mathrm{com}$ maior susceptibilidade à 
infecção pelo HPV. No entanto, alguns autores não encontraram esta relação ${ }^{10}$.

O hábito de fumar tem sido relacionado com a maior incidência e permanência da infecção pelo HPV e de neoplasia pré-invasiva e invasiva ${ }^{11-13}$. A permanência da infecção pelo vírus parece estar relacionada com o desenvolvimento de câncer de colo uterino. No entanto, nas adolescentes, a relação entre o hábito de fumar e a freqüência da infecção pelo HPV é pouca conhecida.

As mulheres com infecções genitais, transmitidas sexualmente ou não, parecem ter maior incidência de infecção pelo HPV. Isto ocorre, provavelmente, pelo aumento da secreção no meio vaginal $^{14}$, que predisporia ao aparecimento de condilomas. A infecção por Candida sp tem sido encontrada em aproximadamente $25 \%$ das pacientes com infecção por HPV ${ }^{15}$.

Trabalhos demonstraram indices mais altos de infecções e lesões neoplásicas não-invasivas nos exames de Papanicolaou de adolescentes em comparação com mulheres adultas ${ }^{16,17}$.

Conforme exposto acima, na adolescência parece haver fenômenos que facilitam a infecção pelo HPV e outros microrganismos. Poucos trabalhos tem estudado a influência da gravidez, do hábito de fumar, dos métodos anticoncepcionais e quais são os achados de microrganismos na citologia cérvico-vaginal nesta faixa etária. Por isto, o objetivo deste trabalho é analisar estes fatores em mulheres com idade inferior a 20 anos com infecção pelo HPV.

\section{Pacientes e Métodos}

Analisamos retrospectivamente 54.985 citologias cérvico-vaginais (coleta tríplice: cervical, ectocervical e endocervical) de pacientes atendidas no Ambulatório de Ginecologia no período de julho de 1993 a dezembro de 1998. Deste total, $6.498(11,8 \%)$ eram de pacientes com idade inferior a 20 anos, sendo que $326(5 \%)$ apresentavam alterações compativeis com infecção pelo HPV, segundo os critérios de Schneider et al. ${ }^{18}$, associada ou não a neoplasia intra-epitelial cervical (NIC) grau I. As pacientes com diagnóstico de NIC II e NIC III em um total de 40 e 7 , respectivamente, foram excluídas. Os critérios de Schneider et al. ${ }^{18}$ são baseados na presença de coilocitose clássica ou, pelo menos, na presença de seis dos nove critérios não-clássicos, quais sejam: coilocitose leve, disqueratose leve, clareamento citoplasmático, grânulos querato-hialinos, estriação citoplasmática, células paraqueratóticas, hipercromasia celular, bi ou multinucleação e halo claro perinuclear.
Foram colhidos dados referentes a: idade, presença de gravidez, uso de algum método contraceptivo regularmente há pelo menos dois meses como ACO, condom, dispositivo intra-uterino (DIU) e laqueadura tubária (TL), hábito de fumar ( $\operatorname{sim}$ ou não) e achados citológicos. Os microrganismos avaliados no exame citológico foram: protozoários (Trichomonas vaginalis), Actinomyces, fungos (Candida sp) e "clue cells". O grupo controle consistiu de pacientes da mesma faixa etária, sem sinais citológicos de infecção pelo HPV, atendidas no mesmo período, selecionadas aleatoriamente com número próximo ao grupo com infecção pelo HPV ( $\mathrm{n}=333$ ). Para análise estatística utilizou-se o teste do $\chi^{2}$ com nível de significância menor que 0,05 .

\section{Resultados}

A idade das adolescentes do grupo com sinais citológicos de infecção pelo HPV variou de 11 a 19 anos $(17,05 \pm 1,8$ anos) e a idade das pacientes do grupo controle variou de 13 a 19 anos $(17,4$ $\pm 1,8$ anos). Observa-se pelos dados da Tabela 1 que a presença da gestação não influenciou no diagnóstico citológico de infecção pelo HPV em adolescentes $(58,9 \%$ versus $55,9 \%$ de gestantes no grupo controle). As adolescentes com infecção pelo HPV eram usuárias de anticoncepcionais orais em $16,9 \%$ dos casos ao passo que $13,8 \%$ do controle utilizavam ACO ( $p<0,01)$, os companheiros de $2,1 \%$ das adolescentes do grupo controle utilizavam condom comparado com $0 \%$ do grupo com infecção pelo HPV $(p<0,01)$. O hábito de fumar não influenciou na incidência de infecção pelo HPV $(21,8 \%$ versus $16,5 \%$ no grupo controle).

$\mathrm{Na}$ Tabela 2, observa-se que o achado de "clue cells" foi o mais freqüente no grupo de adolescentes que apresentavam infecção pelo HPV (22,4\% versus $14,7 \%$ no grupo controle). O grupo das adolescentes com HPV apresentou maior número de citologia sem achado de microrganismos.

\section{Discussão}

Observamos que a gestação não aumentou a freqüência de infecção pelo HPV em adolescentes. Estes resultados divergem de outros trabalhos da literatura que mostram ser a gravidez um fator predisponente à infecção por HPV devido a imunomodulação ou influências hormonais fato comprovado clinicamente pela alta taxa de regressão das lesões após o parto ${ }^{19}$. Entretanto estão de acordo com os achados de de Roda Husman et al. ${ }^{20}$ 
que, estudando a incidência do HPV entre gestantes e não-gestantes com citologia normal pela reação em cadeia da polimerase (PCR), não acharam diferença estatística entre gestantes e nãogestantes com citologias normais. Ressaltamos que, em contraste com os achados de outros autores $^{3,4,13}$, que estudaram mulheres grávidas de diversas idades, o grupo por nós estudado era somente de adolescentes e, talvez, a gravidez não seja um fator predisponente para a infecção pelo HPV nesta faixa etária.

Tabela 1 - Comparação entre os grupos de adolescentes com diagnóstico citológico de infecção por HPV apresentando ou não NIC I (HPV+) e o grupo de adolescentes sem sinais citológicos de infecção por HPV (HPV-) quanto à presença de gravidez, métodos contraceptivos e hábito de fumar.

\begin{tabular}{lrrrr}
\hline & \multicolumn{2}{c}{ HPV + } & \multicolumn{2}{c}{ HPV - } \\
& $\mathbf{n}$ & $\%$ & \multicolumn{1}{c}{$\mathbf{n}$} & $\%$ \\
\hline Gravidez & & & & \\
$\quad$ Sim & 134 & 41,1 & 147 & 44,1 \\
$\quad$ Não & 192 & 58,9 & 186 & 55,9 \\
Método contraceptivo & & & & \\
$\quad$ Anticoncepcional oral * & 55 & 16,9 & 46 & 13,8 \\
Condom * & - & - & 7 & 2,1 \\
Dispositivo intra-uterino & 1 & 0,3 & 4 & 1,2 \\
Laqueadura de trompas & 1 & 0,3 & - & - \\
$\quad$ Nenhum método ou gestantes & 269 & 82,5 & 276 & 82,9 \\
Hábito de fumar & & & & \\
$\quad$ Fumante & 71 & 21,8 & 55 & 16,5 \\
$\quad$ Não Fumante & 229 & 70,2 & 248 & 74,5 \\
$\quad$ Ex-fumante & 8 & 2,5 & 4 & 1,2 \\
$\quad$ Dado não disponivel & 18 & 5,5 & 26 & 7,8 \\
\hline * p<0,01 & & & &
\end{tabular}

Tabela 2 - Comparação dos achados citológicos entre o grupo de adolescentes com diagnóstico citológico de infecção por HPV apresentando ou não NIC I (HPV +) e o grupo de adolescentes sem sinais citológicos de infecção por HPV (HPV -).

\begin{tabular}{lrrrr}
\hline Achado citológico & \multicolumn{2}{c}{ HPV + } & \multicolumn{2}{c}{ HPV - } \\
& n & $\%$ & n & $\%$ \\
\hline Trichomonas vaginallis & 9 & 2,8 & 14 & 4,2 \\
Candida sp* & 51 & 15,6 & 114 & 34,2 \\
"Clue cells"* & 73 & 22,4 & 49 & 14,7 \\
Actinomyces & - & - & - & - \\
Trichomonas vaginalis + Candida sp & 2 & 0,6 & 2 & 0,6 \\
"Clue cells" + Candida sp & 4 & 1,2 & 5 & 1,5 \\
Trichomonas vaginalis + "clue cells" & - & - & 1 & 0,3 \\
Sem achados* & 187 & 57,4 & 148 & 44,5 \\
Total & 326 & 100,0 & 333 & 100,0 \\
\hline${ }^{*}$ p<0,001 & & & &
\end{tabular}

Em relação ao uso de métodos contraceptivos, houve um predomínio do uso de contraceptivos orais nas pacientes adolescentes com infecção pelo HPV em nossos resultados. Dados da literatura mostram que o uso de ACO é um fator de risco para a infecção por $\mathrm{HPV}^{13}$. Outros autores sugerem que o ACO atuaria como co-fator, juntamente com alterações genéticas e alguns tipos de HPV, na transformação de células e na progressão das lesões de baixo para alto grau ${ }^{21}$. Murta et al. ${ }^{22}$ e Moscicki et al. ${ }^{10}$ demonstraram que o uso deste método contraceptivo não é um fator para persistência dessa infecção. Outro questionamento é se as usuárias de ACO poderiam ter maior liberdade sexual com maior número de parceiros sexuais e isto poderia influenciar na maior incidência de infecção pelo HPV. No entanto, este não foi o objetivo deste trabalho, e é um dado que pode ser estudado futuramente.

Entre as adolescentes cujo companheiro é usuário de condom, houve menor incidência de infecção por HPV, sendo o mesmo resultado obtido por Karlsson et al. ${ }^{23}$. A perpetuação da infecção pode ser reduzida com o uso consistente e apropriado, particularmente se as lesões estiverem dentro da proteção do látex ${ }^{24}$. No entanto, outros estudos não demonstraram o efeito protetor do condom ${ }^{9,13,25} \mathrm{e}$ o seu efeito protetor ainda está para ser comprovado.

O fumo tem sido relacionado com infecção por HPV e com neoplasia cervical ${ }^{11,12}$. Isto ocorre provavelmente devido à imunossupressão local causada pelo fumo, que permite com maior facilidade a penetração do vírus nas células ${ }^{26}$. Dados anteriores, por nós publicados ${ }^{13}$, demonstraram que as mulheres fumantes de diferentes faixas etárias apresentam maior incidência de infecção pelo HPV que as não-fumantes. Entretanto, com os resultados deste trabalho, o hábito de fumar parece não ser um fator que influencia na maior freqüência de infecção pelo HPV em adolescentes.

A presença de co-infecções genitais, transmitidas sexualmente ou não, pode ter importância para a proliferação celular associada ao HPV. Isto ocorre, provavelmente, por aumentar a umidade no meio vaginal ${ }^{14}$. É descrito que as mulheres jovens estão mais predispostas a infecções tanto por HPV quanto por outros agentes infecciosos, pela imaturidade biológica da cérvice devido à maior exposição do epitélio colunar da endocérvice ${ }^{27}$.

Nossos resultados mostraram que $34,2 \%$ e $14,7 \%$ das mulheres do grupo controle apresentaram Candida sp e "clue cells", respectivamente, nos exames citológicos, ao passo que no grupo adolescente com HPV, a porcentagem foi de 15,6 e $22,4 \%$. A maior freqüência de "clue cells" em pa- 
cientes com infecção pelo HPV já foi demonstrada em mulheres de várias idades ${ }^{28}$. Este achado é semelhante ao deste trabalho. Como já descrevemos em trabalhos anteriores ${ }^{28}$, é um dado que merece investigação para a melhor compreensão da fisiopatologia da infecção pelo HPV. Mount e Papillo ${ }^{16}$ estudando 10.296 exames de Papanicolaou de mulheres entre 10 e 19 anos, encontraram 7.208 exames normais $(70 \%)$. Os processos infecciosos foram diagnosticados em 1.503 citologias $(14,6 \%)$, sendo 2 casos por Actinomyces, 7 por herpes, 14 por Trichomonas, 699 (6,76\%) por fungos e $784(7,61 \%)$ com desequilíbrio da flora. $\mathrm{E}$ importante ressaltar a diferença entre a freqüência de citologias normais entre o trabalho de Mount e Papillo ${ }^{16}$ e o por nós realizado. Embora estes autores tenham comparado adolescentes com mulheres adultas, e nós somente as adolescentes, o número menor de achados citológicos em adolescentes com infecção pelo HPV é um dado interessante e sugere que a flora vaginal pode influenciar na infecção pelo HPV. Ressaltamos que nossos achados foram baseados em achados citológicos e que a utilização de testes diagnósticos mais sensiveis, como cultura e biologia molecular, podem confirmar nossos resultados.

Baseado em nossos dados concluímos que a infecção pelo HPV é mais freqüente nas usuárias de ACO e com achado citológico de "clue cells" e que os casais usuários de condom têm menor incidência de infecção pelo HPV. A gestação e o hábito de fumar não influenciaram na incidência de infecção pelo HPV.

\section{SUMMARY}

Purpose: to evaluate the influence of pregnancy, habit of smoking, and the contraceptive method in HPV infection and the frequency of cytologic findings in adolescent women with $H P V$ infection.

Methods: a total of 54,985 cytologic examinations of patients seen between July, 1993 and December, 1998 were retrospectively analyzed. Of this total, 6,498 (11.8\%) examinations were from patients under 20 years old. Of the total of 6,498 cytologic examinations, $326(5.9 \%)$ presented signs of HPV infection, with or without grade I cervical intraepithelial neoplasia (CIN). Patients with diagnosis of grade II and III CIN were excluded. The control group consisted of 333 patients paired by age, without cytological signs of HPV infection.

Results: in adolescents, HPV infection was more frequent in oral contraceptive users $(16.9 \%$ versus $13.8 \%, p<0.01)$ and in those who presented with clue cells in cytologic smears
$(22.4 \%$ versus $14.7 \%, p<0.001)$. The frequency of $\mathrm{HPV}$ infection in couples who used condom was $0 \%$ versus $2.1 \%$ in the control group $(p<0.01)$. The difference in the number of pregnant women $(41.1 \%$ versus $44.1 \%)$ and smokers $(21.8 \%$ versus $16.5 \%)$ was not statistically significant.

Conclusions: HPV infection is more frequent in adolescent women in use of oral contraceptive and with clue cells as cytologic finding. HPV infection did not occur in couples who used condom. Gestation and the habit of smoking did not influence the incidence of HPV infection.

KEY WORDS: Human papillomavirus. Adolescents. Cervicovaginal cytology. Contraceptive method.

\section{Agradecimentos}

Ao Serviço de Citologia da Faculdade de Medicina do Triângulo Mineiro e ao Conselho Nacional de Desenvolvimento Científico e Tecnológico (CNPq).

\section{Referências}

1. Derchain SFM, Pinto Neto AM, Oliveira RLC, Santos CC, Pinto e Silva JLC. Infecção por papilomavirus humano e neoplasia intra-epitelial cervical em adolescentes. J Bras Ginecol 1991; 101:499-503.

2. Morrison EA, Ho GY, Vermund SH, et al. Human papillomavirus infection and other risk factors for cervical neoplasia: a case control study. Int J Cancer 1991; 49:6-13.

3. Fife KH, Katz BP, Roush J, Handy VD, Brown DR, Hansell R. Cancer-associated human papillomavirus types are selectively increased in the cervix of women in the first trimester of pregnancy. Am J Obstet Gynecol 1996; 174:148793.

4. Rando RF, Lindheim S, Hasty L, Sedlacek TV, Woodland M, Eder C. Increased frequency of detection of human papillomavirus deoxyribonucleic acid in exfoliated cervical cells during pregnancy. Am J Obstet Gynecol 1989; 161:50-5.

5. Kiviat NB, Koutsky LA, Paavonen JA, et al. Prevalence of genital papillomavirus infection among women attending a college student health clinic or a sexually transmitted disease clinic. J Infect Dis 1989; 159:293-302.

6. Chang-Claude J, Schneider A, Smith E, Blettner M, Wahrendorf J, Tusek L. Longitudinal study of the effects of pregnancy and other factors on detection of HPV. Gynecol Oncol 1996; 60:355-62. 
7. Peng TC, Searle CP $3^{\text {rd }}$, Shah KV, Repke JT, Johnson TR. Prevalence of human papillomavirus infections in term pregnancy. Am J Perinatol 1990; 7:189-92.

8. Smith EM, Johnson SR, Jiang D, et al. The association between pregnancy and human papillomavirus prevalence. Cancer Detect Prev $1991 ; 15: 397-402$.

9. Ley C, Bauer HM, Reingold A, et al. Determinants of genital human papillomavirus infection in young women. J Natl Cancer Inst 1991; 83:997-1003.

10.Moscicki AB, Palefsky J, Gonzales J, Schoolnik GK. Human papillomavirus infection in sexually active adolescent females: prevalence and risk factors. Pediatr Res 1990; 28:507-13.

11.Rohan T, Mann V, MacLaughlin J, et al. PCRdetected genital papillomavirus infection: prevalence and association with risk factors for cervical cancer. Int J Cancer 1991; 49:856-60.

12.Greenberg ER, Vessey M, McPherson K, Yeates D. Cigarette smoking and cancer of the uterine cervix. Br J Cancer 1985; 51:139-41.

13.Murta EFC, Souza MAH, Lombardi W, Borges LS. Aspectos epidemiológicos da infecção pelo papilomavirus humano. J Bras Ginecol 1997; 107:95-9.

14.Oriel JD. The natural history of genital warts. Br J Vener Dis $1971 ; 47: 1-13$.

15.Voog E, Bolmstedt A, Olofsson S, Ryd W, Lowhagen GB. Human papillomavirus infection among women attending an STD clinic correlated to reason for attending, presence for clinical signs, concomitant infection and abnormal cytology. Acta Derm Venereol 1995; 75:75-8.

16. Mount SL, Papillo JL. A study of 10,296 pediatric and adolescent Papanicolaou smear diagnoses in Northern New England. Pediatrics 1999; 103:539-45.

17.Utagawa ML, Pereira SM, Cavaliere MJ, Maeda MY, Shih LW, Shirata NK. Cervical intraepithelial neoplasia in adolescents: study of cytological findings between 1987 and 1995 in São Paulo State - Brazil. Arch Gynecol Obstet 1998; 262:5964.

18.Schneider A, Meinhardt G, De-Villiers EM, Gissmann L. Sensitivity of the cytologic diagnosis of cervical condyloma in comparison with HPVDNA hydridization studies. Diagn Cytopathol $1987 ; 3: 250-5$.

19.Schneider A, Hotz M, Gissmann L. Increased prevalence of human papillomavirus in the lower genital tract of pregnant women. Int $\mathrm{J}$ Cancer 1987; 40:198-201.

20.de Roda Husman AM, Walboomers JM, Hopman E, et al. HPV prevalence in cytomorphologically normal cervical scrapes of pregnant women as determined by PCR: the age-related pattern. J Med Virol 1995; 46:97-102.

21.Pater A, Bayatpour M, Pater MM. Oncogenic transformation by human papillomavirus type 16 deoxyribonucleic acid in the presence of progesterone or progestins from oral contraceptives. Am J Obstet Gynecol 1990; 162:1099-103.

22. Murta EFC, Souza MAH, Adad SJ, et al. Persistência da infecção por papilomavírus humano: análise da idade, sexarca, cor, hábito de fumar e método contraceptivo. J Bras Ginecol 1998;108:117-20.

23.Karlsson R, Jonsson M, Edlund K, et al. Lifetime number of partners as the only independent risk factor for human papillomavirus infection: a population-based study. Sex Transm Dis 1995; 22:119-27.

24.Ferencz Y. Epidemiology and clinical pathophysiology of condylomata acuminata. Am J Obstet Gynecol 1995; 172:1331-9.

25.Kjaer SK, De-Villiers EM, Caglayan H, et al. Human papillomavirus, herpes simplex virus and other potential risk factors for cervical cancer in highrisk area (Greenland) and a low-risk area (Denmark): a second look. Br J Cancer 1993; 67:830-7.

26.Barton SE, Maddox PH, Jenkins D, Edwards R, Cuzick J, Singer A. Effect of cigarette smoking on cervical epithelial immunity: a mechanism for neoplastic change? Lancet 1988; 2:652-4.

27.Koutsky LA, Galloway DA, Holms KK. Epidemiology of genital human papillomavirus infection. Epidemiol Rev 1988; 10:122-63.

28. Murta EFC, Souza MAH, Araújo Júnior E, Adad SJ. Incidence of Gardnerella vaginalis, Candida sp., and human papillomavirus in cytological smears. São Paulo Med J 2000; 118:105-8. 